\title{
Fine Spectrum of the Generalized Difference Operator $B(r, s)$ over the Class of Convergent Series
}

\author{
Amar Jyoti Dutta and Binod Chandra Tripathy \\ Mathematical Sciences Division, Institute of Advanced Study in Science and Technology, Paschim Boragoan, Garchuk, \\ Assam Guwahati 781035, India \\ Correspondence should be addressed to Amar Jyoti Dutta; amar_iasst@yahoo.co.in
}

Received 14 September 2012; Accepted 16 November 2012

Academic Editor: Alexandre Timonov

Copyright (C) 2013 A. J. Dutta and B. C. Tripathy. This is an open access article distributed under the Creative Commons Attribution License, which permits unrestricted use, distribution, and reproduction in any medium, provided the original work is properly cited.

We have determined the spectra of the generalized difference operator $B(r, s)$ over the class of convergent series.

\section{Preliminaries and Background}

Spectral theory is one of the thrust areas of research in mathematical sciences. Due to its usefulness and applicationoriented scope, its importance is not only confined to mathematics but also the theory finds its applications in other fields like aeronautics, electrical engineering, quantum mechanics, structural mechanics and probability theory, ecology, and some others.

Throughout $B(X)$, we denote the set of all bounded linear operators on $X$ into itself. If $T \in B(X)$, where $X$ is a Banach space, then the adjoint operator $T^{*}$ of $T$ is a bounded linear operator on the dual $X^{*}$ of $X$ defined by $\left(T^{*} \phi\right)(x)=\phi(T x)$ for all $\phi \in X^{*}$ and $x \in X$.

Let $T: D(T) \rightarrow X$ be a linear operator defined on $D(T) \subset X$, where $D(T)$ denotes the domain of $T$ and $X$ is a complex normed linear space. For $T \in B(X)$ we associate the operator $(T-\alpha I)$ denoted by $T_{\alpha}$ defined on the same domain $D(T)$, where $\alpha$ is a complex number and $I$ is the identity operator on $D(T)$. The inverse operator $(T-\alpha I)^{-1}$ is denoted by $T_{\alpha}^{-1}$ and known as the resolvent operator of $T$. The resolvent set $\rho(T, X)$ of $T$ is the set of all the regular values $\alpha$ of $T$, such that $T_{\alpha}^{-1}$ exists, bounded and $T_{\alpha}^{-1}$ is defined on a set which is dense in $X$.

Its complement $\mathbb{C} \backslash \rho(T)$ in the complex plane $\mathbb{C}$ is called the spectrum of $T$, denoted by $\sigma(T)$. Thus the spectrum $\sigma(T)$ consists of those values of $\alpha \in \mathbb{C}$, for which $T_{\alpha}$ is not invertible.
The spectrum $\sigma(T)$ is partitioned into three disjoint sets. The point spectrum $\sigma_{p}(T)$ is the set such that $T_{\alpha}^{-1}$ does not exist. Any $\alpha \in \sigma_{p}(T)$ is called the eigen value of $T$. The continuous spectrum $\sigma_{c}(T)$ is the set such that $T_{\alpha}^{-1}$ exists, unbounded and the domain of $T_{\alpha}^{-1}$ is dense in $X$. The residual spectrum $\sigma_{r}(T)$ is the set such that $T_{\alpha}^{-1}$ exists (and may be bounded or not) and the domain of $T_{\alpha}^{-1}$ is not dense in $X$. In finite-dimensional case, continuous spectrum coincides with the residual spectrum, is the empty set and the spectrum consists of only the point spectrum.

Throughout $w, \ell_{\infty}, c, c_{0}$, and $\ell_{p}$ denote the class of all, bounded, convergent, null, and $p$-absolutely summable sequence of fuzzy real or complex terms.

Let $E$ and $F$ be two sequence spaces and $A=\left(a_{n k}\right)$ be an infinite matrix of real or complex numbers $a_{n k}$, where $n$, $k \in N_{0}=\{0,1,2, \ldots\}$. We say that $A$ defines a matrix mapping from $E$ into $F$, denoted by $A: E \rightarrow F$, if for every sequence $x=\left(x_{n}\right) \in E$ the sequence $A x=\left\{(A x)_{n}\right\}$ is in $F$, where $(A x)_{n}=\sum_{k=0}^{\infty} a_{n k} x_{k}(n \in N$ and $x \in E)$, provided the right hand side converges for every $n \in N$ and $x \in E$.

Spectra of some particular types of matrix operators have been investigated from different aspects by Okutoyi [1], Rhoades [2], Tripathy and Saikia [3], Tripathy and Paul [4, 5], and Dutta and Tripathy [6]. Okutoyi [1] studied the spectrum of the Cesàro operator $C_{1}$ on $b v_{0}$. Akhmedov and Başar [7] worked on the spectra of the difference operator $\Delta$ over the sequence space $b v_{p}(1 \leq p<\infty)$. Recently Altay and 
Başar [8] and Furkan et al. [9] determined the spectra of $B(r, s)$ over $c_{0}, c$ and $\ell_{1}, b v$, respectively.

In this paper, we have analyzed the spectra of the generalized difference operator $B(r, s)$ on $\gamma$ of all convergent series. By well-established convention, we define the space $\gamma$ of all "convergent series" by $\gamma=\left\{\left(x_{k}\right) \in w:\left(\sum_{k=0}^{n} x_{k}\right) \in c\right\}$. by

The generalized difference operator $B(r, s)$ is represented

$$
B(r, s)=\left(\begin{array}{ccccc}
r & 0 & 0 & 0 & \cdots \\
s & r & 0 & 0 & \cdots \\
0 & s & r & 0 & \cdots \\
\cdots & \cdots & \cdots & \cdots & \cdots
\end{array}\right) \quad(s \neq 0)
$$

If $T: \gamma \rightarrow \gamma$ is a bounded linear operator with the matrix $A$, then its adjoint operator $T^{*}: \gamma^{*} \rightarrow \gamma^{*}$ is defined by transpose of the matrix $A$ and $\gamma$ is isomorphic to $\ell_{1}$ with the norm $\|x\|=\sum_{k=0}^{\infty}\left|x_{k}\right|$.

The following lemma helps us to determines the norm and to verify that $B(r, s) \in B(\gamma)$.

Lemma 1. The matrix $A=\left(a_{n k}\right)$ gives rise to a bounded linear operator $T \in B\left(\ell_{1}\right)$ from $\ell_{1}$ to itself if and only if the supremum of $\ell_{1}$ norms of the columns of $A$ is bounded.

Lemma 2. $B(r, s): \gamma \rightarrow \gamma$ is a bounded linear operator with $\|B(r, s)\|_{(\gamma, \gamma)} \leq|r|+|s|$.

Proof. It can be easily verified that $B(r, s)$ is linear.

We have

$$
\begin{aligned}
\|B(r, s)\|_{(\gamma, \gamma)} & =\sup _{n}\left|\sum_{k=0}^{n} s x_{k-1}+r x_{k}\right|, \\
& \text { since }\left\|\left(x_{k}\right)\right\|_{c s}=\left|\sum_{k=0}^{n} x_{k}\right| \\
& \leq \sup _{n}|s|\left|\sum_{k=0}^{n} x_{k-1}\right|+\sup _{n}|r|\left|\sum_{k=0}^{n} x_{k}\right| \\
& \leq(|s|+|r|)\|x\|_{\gamma} \\
& \Longrightarrow\|B(r, s)\|_{(\gamma, \gamma)} \leq(|s|+|r|)\|x\|_{\gamma} .
\end{aligned}
$$

Lemma 3 (see [10]). A linear operator $T$ has a dense range if and only if its adjoint operator $T^{*}$ is one to one.

Lemma 4 (see [10]). The adjoint operator $T^{*}$ of $T$ is onto if and only if $T$ has a bounded inverse.

Lemma 5 (see [10]). Let $T \in B(X)$, where $X$ is any Banach space. Then the spectrum of $T^{*}$ is identical with the spectrum of $T$. Furthermore $R_{\lambda}(T)=(T-\lambda I)^{-1}$ and $\rho(T)=\{\lambda \in C$ : $(T-\lambda I)^{-1}$ exist $\}$.

Now we shall determine the spectrum, point spectra, continuous spectra, and residual spectra of the operator $B(r, s)$ on the space $\gamma$.

\section{Main Result}

Theorem 6. $\sigma(B(r, s), \gamma)=\{\alpha \in C:|\alpha-r| \leq s\}$.

Proof. We shall prove this theorem by showing that $(B(r, s)-\alpha I)^{-1}$ exists and is in $B(\gamma)$ for $|\alpha-r|>|s|$ and then show that the operator $(B(r, s), \gamma)$ is not invertible for $|\alpha-r| \leq|s|$.

Let $\alpha$ be such that $|\alpha-r|>|s|$. Since $(B(r, s)-\alpha I)^{-1}$ is a lower triangular matrix, so $(B(r, s)-\alpha I)^{-1}$ exists. Solving $(B(r, s)-\alpha I) x=y$ for $x$ in terms of $y$, we get the following system of equations:

$$
\begin{aligned}
& x_{0}=\frac{1}{r-\alpha} y_{0} \\
& x_{1}=\frac{1}{r-\alpha} y_{1}-\frac{s}{(r-\alpha)^{2}} y_{0} \\
& x_{2}=\frac{1}{r-\alpha} y_{2}-\frac{s}{(r-\alpha)^{2}} y_{1}+\frac{s^{2}}{(r-\alpha)^{3}} y_{0}
\end{aligned}
$$

The $n$th row is given by

$$
x_{n}=\sum_{k \leq n} \frac{(-s)^{n-k}}{(r-\alpha)^{n-k+1}} y_{k} \quad \text { in the } k \text { th place. }
$$

Thus the matrix $\left(a_{n k}\right)$ determined by $(B(r, s)-\alpha I)^{-1}$ is defined by

$$
a_{n k}= \begin{cases}\frac{(-s)^{n-k}}{(r-\alpha)^{n-k+1}}, & \text { for } n \leq k \\ 0, & \text { otherwise }\end{cases}
$$

We observe that

$$
\begin{aligned}
& \|B(r, s)-\alpha I\|_{(\gamma, \gamma)}^{-1} \\
& =\sup _{n} \sum_{k=0}^{n}\left|\frac{(-s)^{n-k}}{(r-\alpha)^{n-k+1}}\right| \\
& =\left|\frac{1}{r-\alpha}\right| \sup _{n} \sum_{k=0}^{n}\left|\frac{s}{(r-\alpha)}\right|^{n-k} \\
& =\left|\frac{1}{r-\alpha}\right| \sum_{k=0}^{n}\left|\frac{s}{(r-\alpha)}\right|^{n}<\infty .
\end{aligned}
$$

Thus $(B(r, s)-\alpha I)^{-1} \in B(\gamma)$.

Now we show that the operator $(B(r, s)-\alpha I)$ is not invertible for $|\alpha-r| \leq|s|$.

Let $\alpha$ be such that $|\alpha-r| \leq|s|$ and $\alpha \neq r$. Since $(B(r, s)-$ $\alpha I)$ is triangular, so $(B(r, s)-\alpha I)^{-1}$ exists. From (6) we have $\|B(r, s)-\alpha I\|_{(\gamma, \gamma)}^{-1}=\infty$, whenever $|\alpha-r| \leq|s|$, that is 
$(B(r, s)-\alpha I)^{-1} \notin B(\gamma)$. When $\alpha=r$, the operator $(B(r, s)-$ $\alpha I)$ is represented by the matrix $B(s)$ given by

$$
B(s)=\left[\begin{array}{cccc}
0 & 0 & 0 & \cdots \\
s & 0 & 0 & \cdots \\
0 & s & 0 & \ldots \\
\cdots & \cdots & \ldots & \ldots
\end{array}\right] .
$$

We observe that $(B(s)-s I) x=\theta \Rightarrow x=\theta$. Thus $(B(s)-s I)$ : $\gamma \rightarrow \gamma$ is injective but its range is not a dense set. Hence $(B(s)-s I)$ is not invertible. This shows that $(B(r, s)-\alpha I)$ is not invertible for $|\alpha-r| \leq|s|$. This completes the proof.

Theorem 7. $\sigma_{p}(B(r, s), \gamma)=\varnothing$.

Proof. Let $B(r, s) x=\alpha x$, for $x \neq \theta=(0,0,0, \ldots)$ in $\gamma$. We have the following system of equations:

$$
\begin{gathered}
r x_{0}=\alpha x_{0} \\
s x_{0}+r x_{1}=\alpha x_{1} \\
\vdots \\
s x_{k}+r x_{k+1}=\alpha x_{k+1} \\
\vdots \\
\vdots
\end{gathered}
$$

If $x_{i}$ is the first nonzero term of the sequence $x=\left(x_{n}\right), r=\alpha$, hence we have

$$
\begin{aligned}
& s x_{i}+r x_{i+1}=\alpha x_{i+1} \\
& \Longrightarrow s x_{i}=0 \Longrightarrow x_{i}=0, \quad \text { since } s \neq 0 .
\end{aligned}
$$

This contradicts to the fact that $x_{i} \neq 0$.

Thus $(B(r, s)-\alpha I) x=\theta \Rightarrow x=\theta$ and hence $(B(r, s)-\alpha I)$ : $\gamma \rightarrow \gamma$ is injective.

That is, $\sigma_{p}(B(r, s), \gamma)=\varnothing$.

Theorem 8. $\sigma_{p}\left(B(r, s)^{*}, \gamma^{*}\right)=\{\alpha \in C:|\alpha-r|<s\}$.

Proof. Suppose that $B(r, s)^{*} x=\alpha x$, for $x(\neq \theta) \in \gamma^{*} \cong \ell_{1}$. Now we have the following system of equations:

$$
\begin{gathered}
r x_{0}+s x_{1}=\alpha x_{0} \\
r x_{1}+s x_{2}=\alpha x_{1} \\
\vdots \\
r x_{k}+s x_{k+1}=\alpha x_{k}
\end{gathered}
$$

Solving the above systems of linear equations, we get

$$
x_{n}=\left(\frac{\alpha-r}{s}\right)^{n} x_{0}, \quad \text { for } n \in N .
$$

We observe that $x=\left(x_{n}\right) \in \gamma^{*}$ if and only if $|\alpha-r|<s$.

This completes the proof.
Theorem 9. $\sigma_{r}(B(r, s), \gamma)=\{\alpha \in C:|\alpha-r|<s\}$.

Proof. We prove that the range of $(B(r, s)-\alpha I)$ is not dense in $\gamma$ but $(B(r, s)-\alpha I)$ has an inverse, bounded, or unbounded.

Theorem 8 implies that $\left(B(r, s)^{*}-\alpha I\right)$ is not one-to-one and therefore by Lemma $3,(B(r, s)-\alpha I)$ is not dense in $\gamma$. We have also seen that $(B(r, s)-\alpha I)^{-1}$ exist and $\overline{(B(r, s)-\alpha I)} \neq \gamma$ for those $\alpha$ satisfying $|\alpha-r|<s$. Again for $\alpha \neq s,(B(r, s)-\alpha I)$ is triangular and therefore has an inverse.

This completes the proof.

Theorem 10. $\sigma_{c}(B(r, s), \gamma)=\{\alpha \in C:|\alpha-r|=s\}$.

Proof. It is well known that $\sigma(B(r, s), \gamma)$ is the disjoint union of $\sigma_{p}(B(r, s), \gamma), \sigma_{r}(B(r, s), \gamma)$, and $\sigma_{c}(B(r, s), \gamma)$ since $\sigma_{p}(B(r, s), \gamma)=\varnothing$ and $\sigma_{r}(B(r, s), \gamma)=\{\alpha \in C:|\alpha-r|<s\}$.

Thus $\sigma_{c}(B(r, s), \gamma)=\{\alpha \in C:|\alpha-r|=s\}$.

This completes the proof.

\section{Conclusion}

In this paper, the spectra of matrix operator on convergent series is established. In a similar way the spectra of other matrix operators can also be determined over the convergent series.

\section{Acknowledgments}

The authors thank the reviewer for his comments on the paper; those improved the presentation of the paper. The work of the authors is financially supported by the Council of Scientific and Industrial Research, India vide Grant no. 25(0182)/10/EMR-II.

\section{References}

[1] J. I. Okutoyi, "On the spectrum of $C_{1}$ as an operator on $b v_{0}$," Australian Mathematical Society Journal A, vol. 48, no. 1, pp. 79-86, 1990.

[2] B. E. Rhoades, "The fine spectra for weighted mean operators," Pacific Journal of Mathematics, vol. 104, no. 1, pp. 219-230, 1983.

[3] B. C. Tripathy and P. Saikia, "On the spectrum of the Cesáro operator $C_{1}$ on $\overline{b v} \cap \ell_{\infty}$," Mathematica Slovaca. In press.

[4] B. C. Tripathy and A. Paul, "The Spectrum of the operator $D(r, 0$, $0, s)$ over the Sequence Space $c_{0}$ and $c$," Kyungpook Mathematical Journal. In press.

[5] B. C. Tripathy and A. Paul, "Spectra of the operator $B(f, g)$ on the vector valued sequence space $c_{0}(X)$," Boletim da Sociedade Paranaense de Matemática, vol. 31, no. 1, pp. 105-111, 2013.

[6] A. J. Dutta and B. C. Tripathy, "Fine spectra of upper triangular double band matrices over the sequence spaces $l_{1}$ and $b v$," Applied Sciences. In press.

[7] A. M. Akhmedov and F. Başar, "The fine spectra of the Cesàro operator $C_{1}$ over the sequence space $b v_{p}(1 \leq p<$ $\infty)$," Mathematical Journal of Okayama University, vol. 50, pp. 135-147, 2008.

[8] B. Altay and F. Başar, "On the fine spectrum of the generalized difference operator $B(r, s)$ over the sequence spaces $c_{0}$ 
and c," International Journal of Mathematics and Mathematical Sciences, vol. 2005, no. 18, pp. 3005-3013, 2005.

[9] H. Furkan, H. Bilgiç, and K. Kayaduman, "On the fine spectrum of the generalized difference operator $B(r, s)$ over the sequence spaces $l_{1}$ and $b v$," Hokkaido Mathematical Journal, vol. 35, no. 4 , pp. 893-904, 2006.

[10] S. Goldberg, Unbounded Linear Operators, Dover Publications, New York, NY, USA, 1985. 


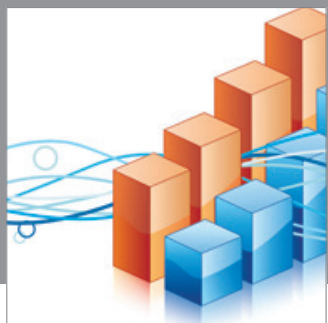

Advances in

Operations Research

mansans

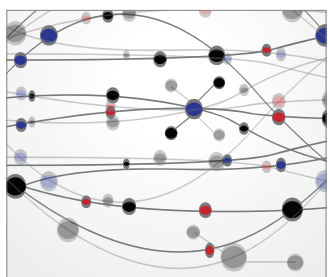

The Scientific World Journal
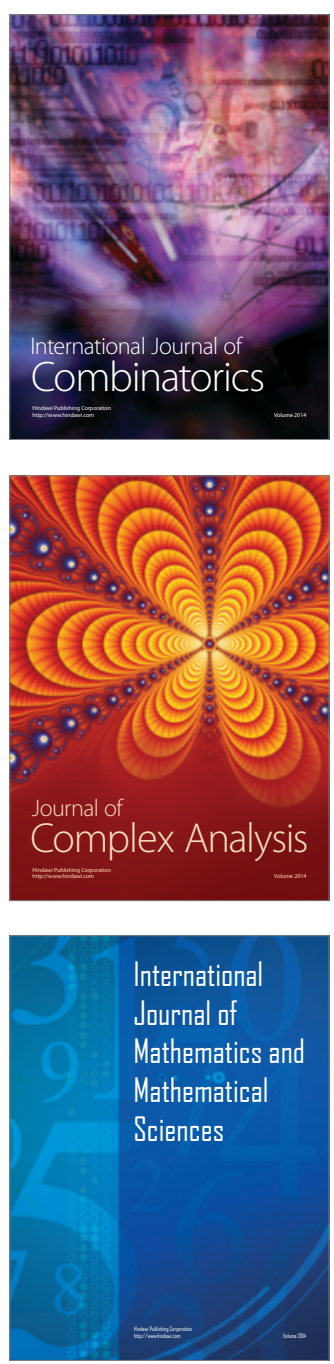
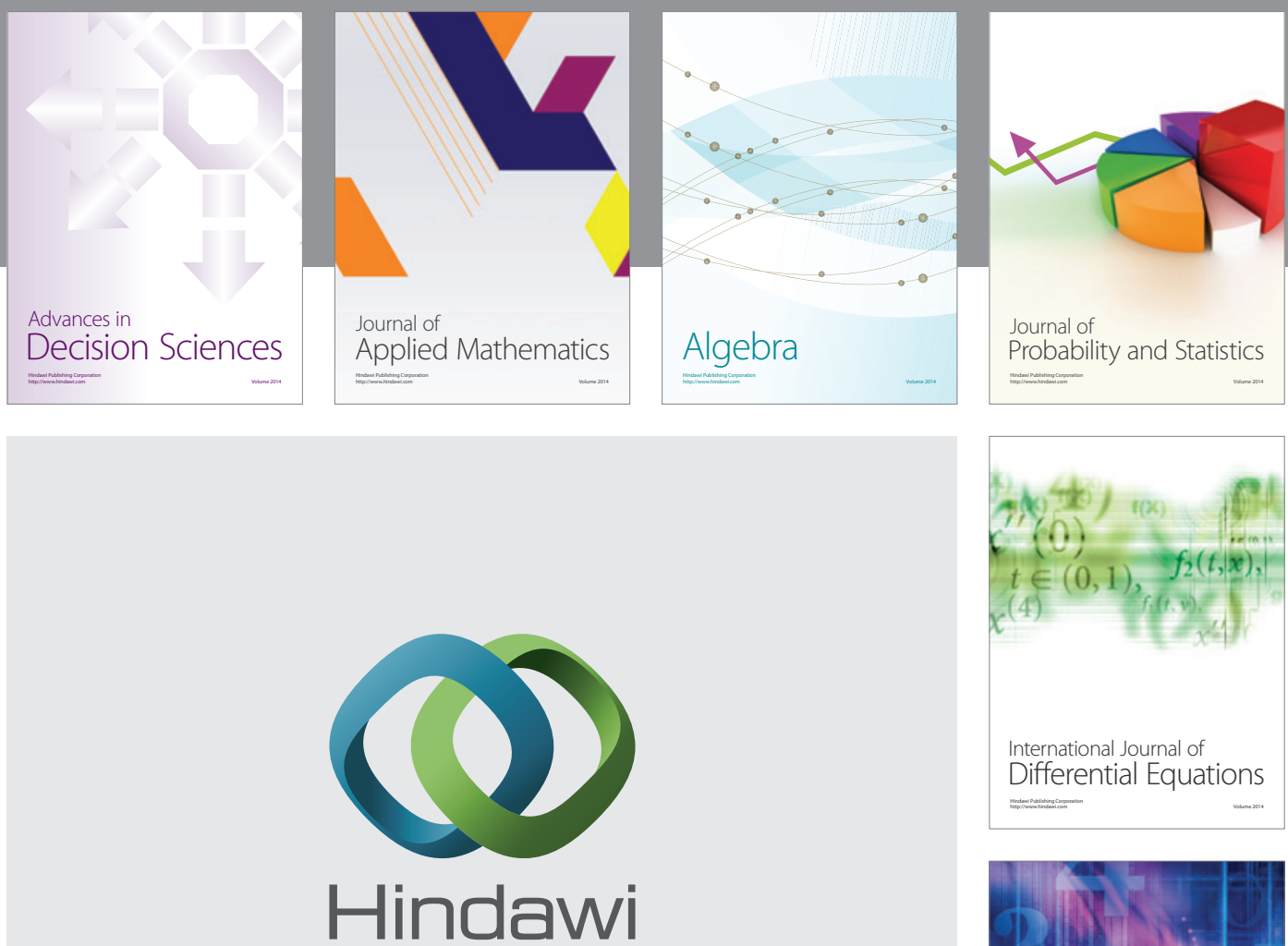

Submit your manuscripts at http://www.hindawi.com
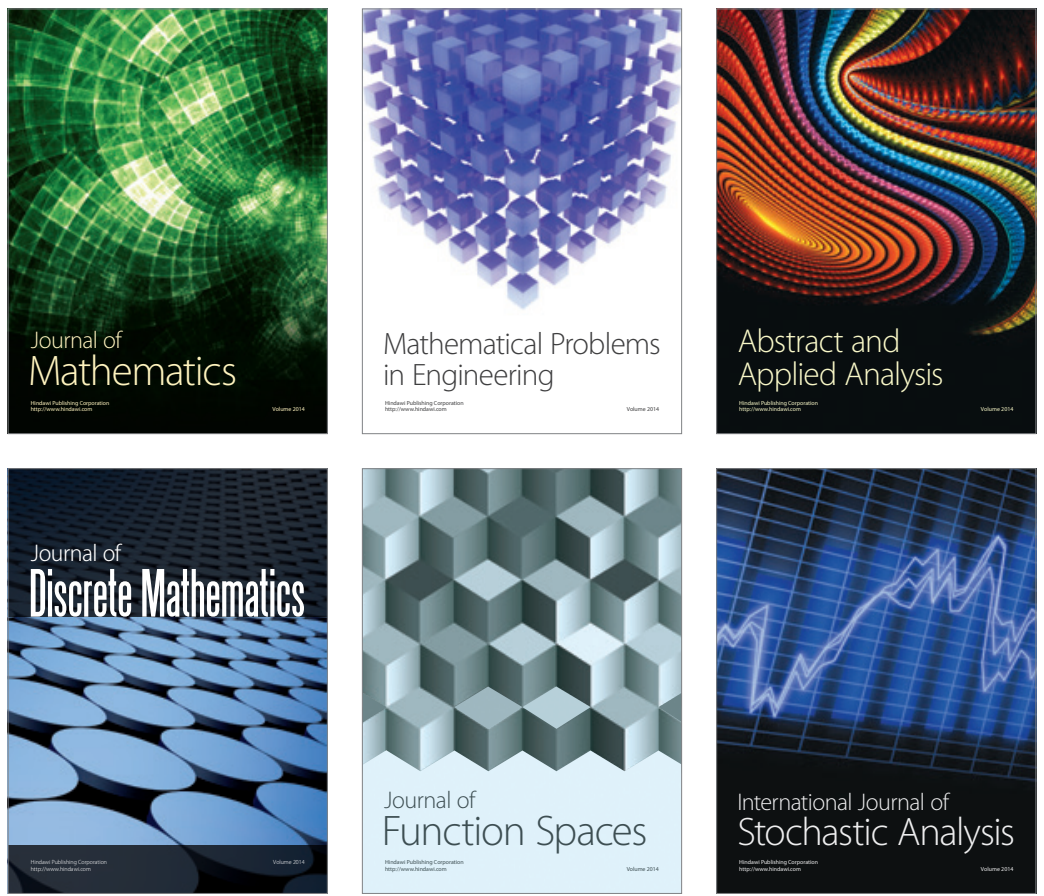

Journal of

Function Spaces

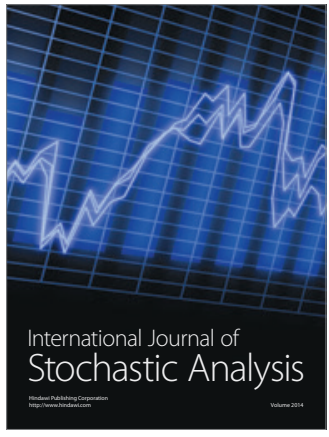

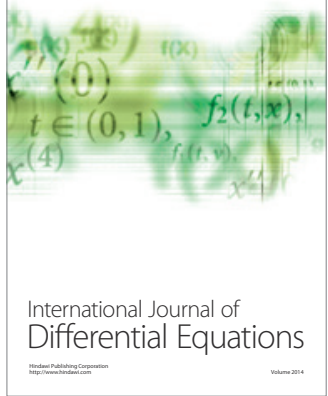
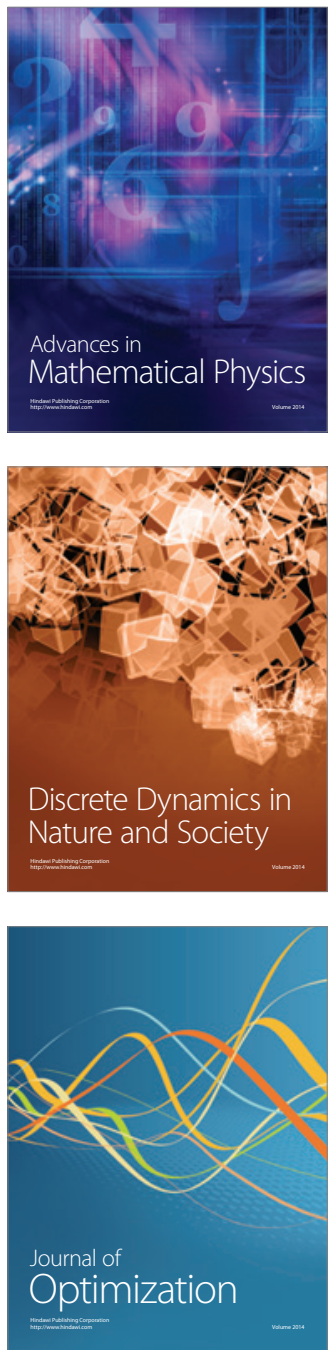